\title{
Axisymmetric vortex breakdown Part 2. Physical mechanisms
}

\author{
By G. L. BROWN AND J. M. LOPEZ \\ Aeronautical Research Laboratory, P.O. Box 4331, Melbourne, Vic., 3001, Australia
}

(Received 11 February 1988 and in revised form 14 May 1990)

The physical mechanisms for vortex breakdown which, it is proposed here, rely on the production of a negative azimuthal component of vorticity, are elucidated with the aid of a simple, steady, inviscid, axisymmetric equation of motion. Most studies of vortex breakdown use as a starting point an equation for the azimuthal vorticity (Squire 1960), but a departure in the present study is that it is explored directly and not through perturbations of an initial stream function. The inviscid equation of motion that is derived leads to a criterion for vortex breakdown based on the generation of negative azimuthal vorticity on some stream surfaces. Inviscid predictions are tested against results from numerical calculations of the NavierStokes equations for which breakdown occurs.

\section{Introduction}

The attraction of the confined swirling flow of Part 1 (Lopez 1990; see also Lopez 1988) for a study of vortex breakdown is that the flow is defined by only four variables, $H, R, \Omega$ and $\nu$ (respectively the height and radius of the cylinder, angular velocity and kinematic viscosity), each of which, in an experiment, can be very accurately determined. Similarly, from a numerical point of view, the flow is confined in a fixed volume with very well-defined boundary conditions. It was hoped that if numerical solutions for various $H / R$ and Reynolds number $R e=\Omega R^{2} / \nu$ could be obtained which very accurately predicted the resulting flow in such a well-defined experiment then the physical mechanisms of vortex breakdown could be elucidated from these solutions and, further, that these mechanisms might be generalized to the swirling flows of more practical significance in pipes and in a free stream.

Many features of vortex breakdown have been well recognized and the early works of Squire (1960) and Benjamin (1962) have been the starting point for many subsequent studies. These have led to postulated criteria for vortex breakdown based on the ratio of an axial to an azimuthal component of velocity (e.g. Squire 1960; Benjamin 1962; Keller, Egli \& Exley 1985; and Spall, Gatski \& Gresch 1987). A departure in the present study from these formulations is that the numerical solutions are explored not from a consideration of perturbations to an initial stream function but more directly from the development of the azimuthal component of vorticity. This leads to a criterion for a recirculation region based on the relationship between the angle of the velocity vector and the vorticity vector on stream surfaces upstream of this region.

The numerical results in Part 1 demonstrate the accuracy with which the experimental results can be predicted for confined flows in which 'vortex breakdown' develops and this paper, Part 2, explores the underlying physical mechanisms which 
account for the essential features of the flow and are important in 'vortex breakdown'.

\section{Confined swirling flows}

\subsection{Summary of the principal features of the flow}

As suggested in Part 1, for the confined flow in which $H / R=2.5$ and $R e>1600$, the central core flow, i.e. the flow returning towards the rotating endwall, might be regarded as practically inviscid apart from the recirculation zone and the fluid that passes near to it. This first approximation, expected to be increasingly valid as Reynolds number increases, is supported by figures $2(f)$ and 3 from Part 1 which show contours of the stream function $\psi$, the angular momentum, or circulation, $\Gamma$ and the total head $\mathscr{H}$. A detailed comparison between these contours shows that in this central core region $\Gamma$ and $\mathscr{H}$ are approximately constant on stream surfaces, apart from those surfaces within and near to the recirculation bubble where $\Gamma$ and $\mathscr{H}$ are relatively small. An approximation that they are constant on the stream surface would seem unlikely to change the principal features of the flow. Of course, the experiments and the numerical calculations show that this core flow is critically dependent on Reynolds number but mainly in the sense, it seems, that $R e$ determines an 'upstream' distribution of $\Gamma(r)$ and $\mathscr{H}(r)$ and that once this distribution is established the subsequent core flow, in which vortex breakdown occurs, is largely an inviscid rotational flow apart from the flow within and near to the recirculation zones.

The most striking features of this core flow are the divergence from an upstream narrow core to a much larger diameter flow which is almost in solid-body rotation, and correspondingly, the development of a wave as a result of an 'overshoot' in the initial divergence. The apparent effect of increasing Reynolds number is to change the upstream distribution of $\Gamma(r)$ and $\mathscr{H}(r)$ in such a way as to increase the initial divergence, increase the 'overshoot', reduce the wavelength of the resulting wave and reduce the 'damping' of this wave. The contours of the stream function (figure 2 from Part 1) suggest that the appearance of recirculation bubbles is related to the waviness in the outer flow. These preliminary observations provide useful approximations and working hypotheses which are explored in the remainder of $\$ 2$.

\subsection{Theoretical considerations}

Steady, inviscid, axisymmetric swirling flow is particularly interesting in that it can be looked at from the point of view of an interaction between the total head and the angular momentum of the fluid (both of which are conserved on a stream surface), or in terms of a balance between the radial pressure gradient and the centrifugal force, or in terms of the generation of the azimuthal component of vorticity through the stretching and tilting of vortex lines.

The relationship between these three different perspectives is as follows. In terms of the radial pressure gradient and the centrifugal force, the other radial acceleration terms are

$$
u \frac{\partial u}{\partial r}+w \frac{\partial u}{\partial z}=\frac{v^{2}}{r}-\frac{1}{\rho} \frac{\partial p}{\partial r}
$$


In terms of the angular momentum (or circulation) $\Gamma=r v$, and the total head $\mathscr{H}=$ $p / \rho+\frac{1}{2}\left(u^{2}+v^{2}+w^{2}\right)$, (1) may be written as

$$
w \frac{\partial u}{\partial z}=\frac{\Gamma}{r^{2}} \frac{\partial \Gamma}{\partial r}-\frac{\partial \mathscr{H}}{\partial r}+w \frac{\partial w}{\partial r}
$$

Thirdly, since the azimuthal component of vorticity is $\eta=\partial u / \partial z-\partial w / \partial r$, then from (2)

$$
w \eta=\frac{\Gamma}{r^{2}} \frac{\partial \Gamma}{\partial r}-\frac{\partial \mathscr{H}}{\partial r}
$$

or, since the stream function $\psi$ is determined by $u=-(1 / r)(\partial \psi / \partial z)$ and $w=$ $(1 / r)(\partial \psi / \partial r)$ and since $\Gamma$ and $\mathscr{H}$ are constant on $\psi$, it follows from (3) that

$$
\eta=\frac{\Gamma}{r} \frac{\mathrm{d} \Gamma}{\mathrm{d} \psi}-r \frac{\mathrm{d} \mathscr{H}}{\mathrm{d} \psi} .
$$

Equation (4) has been a starting point for most discussions of vortex breakdown. Following Squire (1960), it is usually rewritten by replacing $\eta$ with $-(1 / r) \nabla^{2} \psi$. Notwithstanding that $\Gamma$ and $\mathscr{H}$ are functions of $r$ and $z$, the equation as it stands is of such a simple form that we have pursued its implications more directly. In particular, if the curve $C$ in the $(r, z)$-plane whose radius $r$ is given by $r=\sigma(z)$ is chosen such that on $C$ the stream function is a constant, i.e. $\psi(r, z)=\psi_{1}$, then on $C$

or, on a stream surface

$$
\begin{gathered}
\eta(z)=\frac{\Gamma\left(\psi_{1}\right)}{\sigma} \Gamma\left(\psi_{1}\right)-\sigma \mathscr{H}^{\prime}\left(\psi_{1}\right) \\
\mathrm{d} \psi=0 \quad \text { and } \quad u=w \sigma^{\prime}(z)
\end{gathered}
$$

$$
\eta(z)=\frac{A}{\sigma}-B \sigma
$$

and

$$
\mathrm{d} \eta=-\left(\frac{A}{\sigma^{2}}+B\right) \mathrm{d} \sigma,
$$

where $A=\Gamma\left(\psi_{1}\right) \Gamma^{\prime}\left(\psi_{1}\right)$ and $B=\mathscr{H}^{\prime}\left(\psi_{1}\right)$.

Equation (7) is remarkable since it provides such a simple expression for one component of the vorticity in terms only of the radius of the stream surface yet in a complex flow where the vorticity is three-dimensional, not normal to the velocity, and where the stretching of vorticity is an essential mechanism in the flow.

Further insight into the implications of (7) and (8) is gained if it is assumed that at some upstream station $z_{0}$ and on a particular stream surface $\psi=\psi_{1}$, the radius of the stream surface is $\sigma_{0}$ and the azimuthal and axial components of both the velocity, i.e. $v$ and $w$, and vorticity, i.e. $\eta$ and $\zeta$, are known and have the values $v_{0}, w_{0}, \eta_{0}$ and $\zeta_{0}$ (see figure 1). In this case $A$ and $B$ may be determined simply, since

$$
r \zeta=\frac{\partial \Gamma}{\partial r}=r w \frac{\mathrm{d} \Gamma}{\mathrm{d} \psi},
$$

so that on $\psi_{1}$

$$
\Gamma^{\prime}\left(\psi_{1}\right)=\frac{\zeta_{0}}{w_{0}}
$$




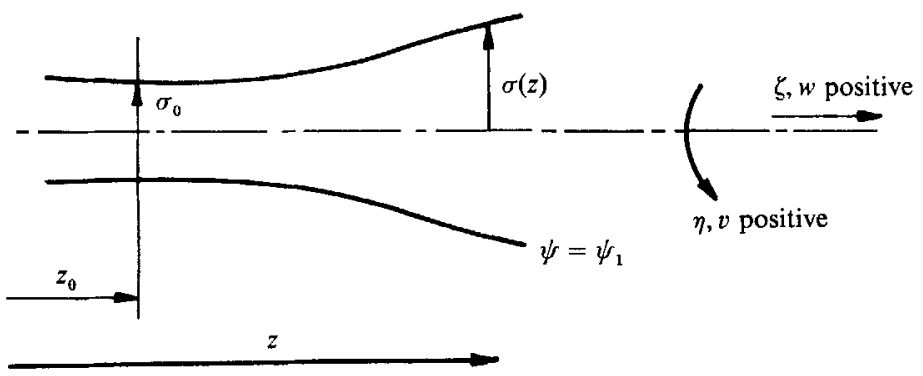

at $z=z_{0}, r=\sigma_{0}\left(\text { if } \frac{\partial u}{\partial z}=0 \text { then } \eta_{0}=-\frac{\partial w}{\partial r}\right)_{\sigma_{0}}$
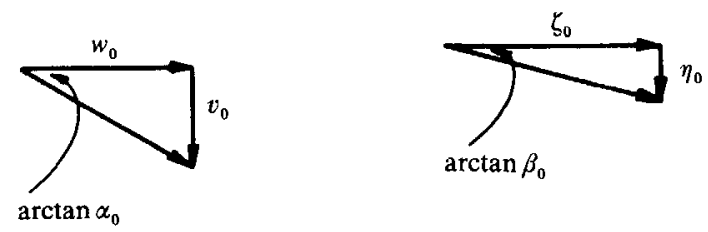

Figure 1. Coordinate system used and a typical stream surface $\psi=\psi_{1}$ for which $r=\sigma(z)$ and the helix angles for the velocity and vorticity at $z_{0}$ are $\alpha_{0}$ and $\beta_{0}$.

and

$$
A=\Gamma\left(\psi_{1}\right) \Gamma^{\prime}\left(\psi_{1}\right)=\frac{\sigma_{0} v_{0}}{w_{0}} \zeta_{0}
$$

$B$ may be determined from (7) evaluated at $z=z_{0}$. If $\eta_{0} \neq 0$, then

$$
\begin{gathered}
B=\frac{\eta_{0}}{\sigma_{0}}\left(\frac{v_{0} \zeta_{0}}{w_{0} \eta_{0}}-1\right), \\
B=\frac{v_{0} \zeta_{0}}{\sigma_{0} w_{0}} .
\end{gathered}
$$

and if $\eta_{0}=0$ then

Thus from (7) the equation for $\eta$ on the stream surface downstream from $z_{0}$ is

or

$$
\begin{gathered}
\eta=\alpha_{0} \zeta_{0}\left(\frac{\sigma_{0}}{\sigma}-\frac{\sigma}{\sigma_{0}}\right) \text { for } \eta_{0}=0 \\
\frac{\eta}{\eta_{0}}=\frac{\sigma_{0}}{\sigma}\left(\frac{\alpha_{0}}{\beta_{0}}\right)-\frac{\sigma}{\sigma_{0}}\left(\frac{\alpha_{0}}{\beta_{0}}-1\right) \text { for } \eta_{0} \neq 0,
\end{gathered}
$$

where $\alpha_{0}=v_{0} / w_{0}$ and $\beta_{0}=\eta_{0} / \zeta_{0}$ are the tangents of the helix angle for the velocity and vorticity respectively. Thus, downstream of $z_{0}$, the ratio of $\eta$ on a stream surface to its value on the surface at $z_{0}$ depends only on the ratio of the tangents of the helix angles of the vorticity and velocity on the surface at $z_{0}$ and on the ratio of the radius of the stream surface to the radius at $z_{0}$.

From the point of view of vortex breakdown, (7) and (9) have important implications. A reasonable hypothesis (in the absence of bodies in the flow field) is that the development of a negative azimuthal component of vorticity on some stream surfaces is necessary if the axial velocity is to be brought to zero (see figure 


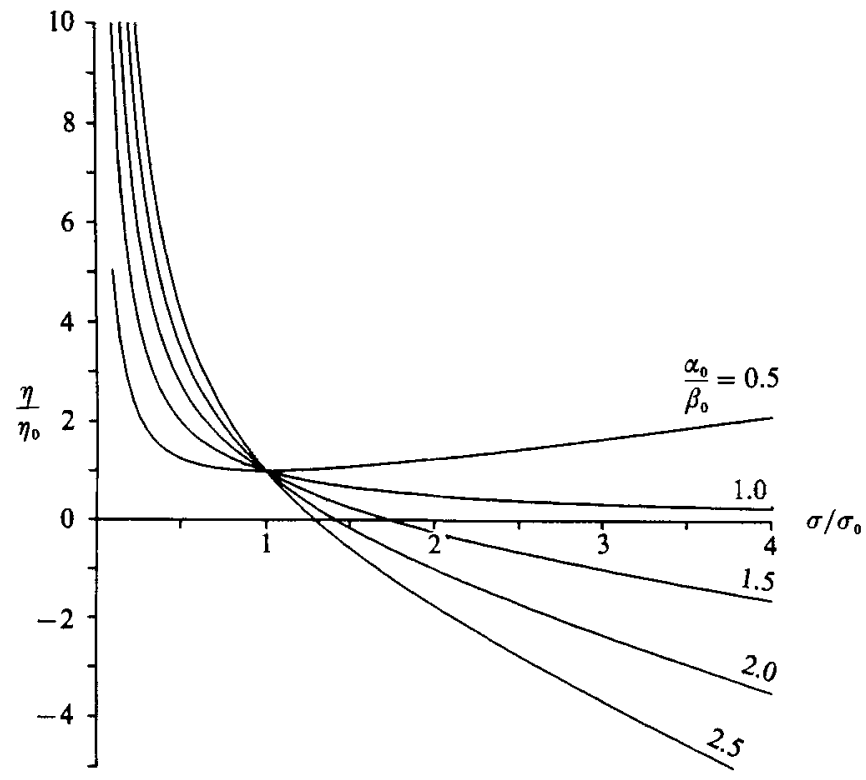

Figure 2. Plot of $\eta / \eta_{0}=\left(\sigma_{0} / \sigma\right)\left(\alpha_{0} / \beta_{0}\right)-\left(\sigma / \sigma_{0}\right)\left(\alpha_{0} / \beta_{0}-1\right)$ (equation (9)) for various values of $\alpha_{0} / \beta_{0}$ as indicated.

1). $\uparrow$ This is intuitively reasonable because the velocity due to a field of vorticity is given by

$$
U(r)=-\frac{1}{4 \pi} \int \frac{s \times \omega\left(r^{\prime}\right)}{s^{3}} \mathrm{~d} V,
$$

where $s=r-r^{\prime}$, and in the case of an axisymmetric flow field the velocity on the axis due to the vorticity field is

$$
w(0, z)=\frac{1}{2} \int_{-\infty}^{\infty} \int_{0}^{\infty} \frac{\sigma^{2} \eta\left(\sigma, z^{\prime}\right)}{\left[\sigma^{2}+\left(z-z^{\prime}\right)^{2}\right]^{\frac{3}{2}}} \mathrm{~d} \sigma \mathrm{d} z^{\prime} .
$$

We infer that, in addition to any irrotational component of the velocity which satisfies the boundary conditions, it is the local azimuthal vorticity which contributes most to the velocity at a point on the axis since the contribution from vorticity in the far field decays as $\left(z-z^{\prime}\right)^{3}$ and, importantly, the velocity on the axis due to the vorticity will only be zero or negative at some $z$ if $\eta\left(\sigma, z^{\prime}\right)$ is negative in some region since the other terms in the integral are everywhere positive.

Equation (7) implies that $\eta$ can only become negative if $B$ is positive or, more specifically from (9), for $\eta_{0}$ positive, $\eta$ will only become negative on a diverging stream surface if $\alpha_{0}>\beta_{0}$, i.e. $v_{0} / w_{0}>\eta_{0} / \zeta_{0}$. A helix angle for the velocity which exceeds that of the vorticity on some stream surfaces would seem therefore to be a necessary condition for vortex breakdown to occur since, from (9), as $\sigma / \sigma_{0}$ increases from unity, then for $\alpha_{0}>\beta_{0}, \eta / \eta_{0}$ decreases to zero and becomes negative at a sufficiently large $\sigma / \sigma_{0}$. Figure 2 illustrates the behaviour and the dependence on $\alpha_{0} / \beta_{0}$. (In $\$ 3.2$ the development of $\eta / \eta_{0}$ on a stream surface obtained from the

$\dagger$ For simplicity, in this paper $z$ is taken to increase in the direction of the primary axial velocity in the core flow and correspondingly negative azimuthal vorticity will induce an axial velocity in the opposite direction. In Part 1 increasing $z$ is in the opposite direction, away from the rotating endwall, and the sign of the corresponding azimuthal vorticity is the reverse of its sign in this paper. 
numerical solutions of the Navier-Stokes equations for a swirling flow is compared with the inviscid prediction from (9) and is found to be in good agreement.)

The relatively rapid divergence of stream surfaces in the region of breakdown can also be qualitatively understood from these equations. The development of negative $\eta$ on stream surfaces will induce a negative axial velocity on the axis which, by continuity, will lead to a further increase in $\sigma$ and correspondingly (from (7) or (9)), a further increase in negative vorticity, etc. This can be thought of as a kind of 'positive feedback', but in essence, it is a matter of compatibility between the velocity $(U)$ and the vorticity $(\nabla \times U)$ arrived at from a consideration of the dynamics of vorticity on a stream surface on the one hand and the requirements of continuity on the other.

We are led to conclude that, in the absence of viscous or turbulent diffusion, a necessary condition for vortex breakdown to occur downstream of $z_{0}$ would seem to be that at $z_{0}, v_{0} / w_{0}>\eta_{0} / \zeta_{0}$ on some stream surfaces.

A reduction in azimuthal vorticity with increasing radius is readily understood physically for the simple case of a flow that is cylindrical upstream and has $\eta=0$. If two material points on a vortex line (which is axial in this cylindrical flow) move with the fluid and the leading point advances to a location where the radius has begun to increase, then this leading point will experience a reduction in azimuthal velocity due to the conservation of $\Gamma$ on the stream surface. The material line between the points is therefore stretched and tilted, and since the vorticity moves with this material line, a negative azimuthal component of vorticity is generated. This accords of course with the $\eta_{0}=0$ case of equation (9). More generally, however, for $\eta_{0} \neq 0$ then from (8) or $(9)$,

$$
\frac{\mathrm{d} \eta}{\mathrm{d} \sigma}=\frac{-\eta_{0}}{\sigma_{0}}\left(\left(\frac{\sigma_{0}^{2}}{\sigma^{2}}+1\right) \frac{\alpha_{0}}{\beta_{0}}-1\right) .
$$

It follows that for $\sigma / \sigma_{0} \geqslant 1$ and $\eta_{0}$ positive then for $\alpha_{0} / \beta_{0}<0.5, \mathrm{~d} \eta / \mathrm{d} \sigma>0$; for $0.5<\sigma_{0} / \beta_{0}<1, \eta$ remains positive and at sufficiently large $\sigma / \sigma_{0}, \mathrm{~d} \eta / \mathrm{d} \sigma>0$; and from (9) only for $\alpha_{0} / \beta_{0}>1$ does $\eta$ become negative for sufficiently large $\sigma / \sigma_{0}$ and $\mathrm{d} \eta / \mathrm{d} \sigma$ remain negative (see figure 2). The result that $\mathrm{d} \eta / \mathrm{d} \sigma>0$ for $\beta_{0}>2 \alpha_{0}$ seems a counter-intuitive one at first sight and in terms of the above 'feedback' argument, suggests a kind of stability in the sense that a perturbation in $\sigma$ would generate azimuthal vorticity which, by continuity, would produce a restoring change in $\sigma$. We have not pursued the possible connection between these results and the supercritical and subcritical ideas which, following Benjamin (1962), have been associated with vortex breakdown.

With these general considerations in mind, the vortex breakdown features of the confined swirling flow may be interpreted as follows.

\subsection{Physical mechanisms}

The summary in $\$ 2.1$ and these theoretical considerations suggest that an important question to ask is why the strong vortical core flow begins to diverge? A simple, if simplistic, answer is clear from (1). Downstream from the point where $u=0$ and the stream surface has its smallest radius (or, more generally, downstream from a region in which the flow is cylindrical) there will be divergence, i.e. positive $\partial u / \partial z$ only if the centrifugal force exceeds the radial pressure gradient (in the absence of viscous stresses). For the confined flow, this imbalance clearly arises from the fact that the radial distribution of $\Gamma$ and $\mathscr{H}$ in the narrow upstream core region is determined by the 'upstream' history of a fluid particle as it travels on its closed stream surface. In particular, the particle acquires and loses most of its angular momentum and total 


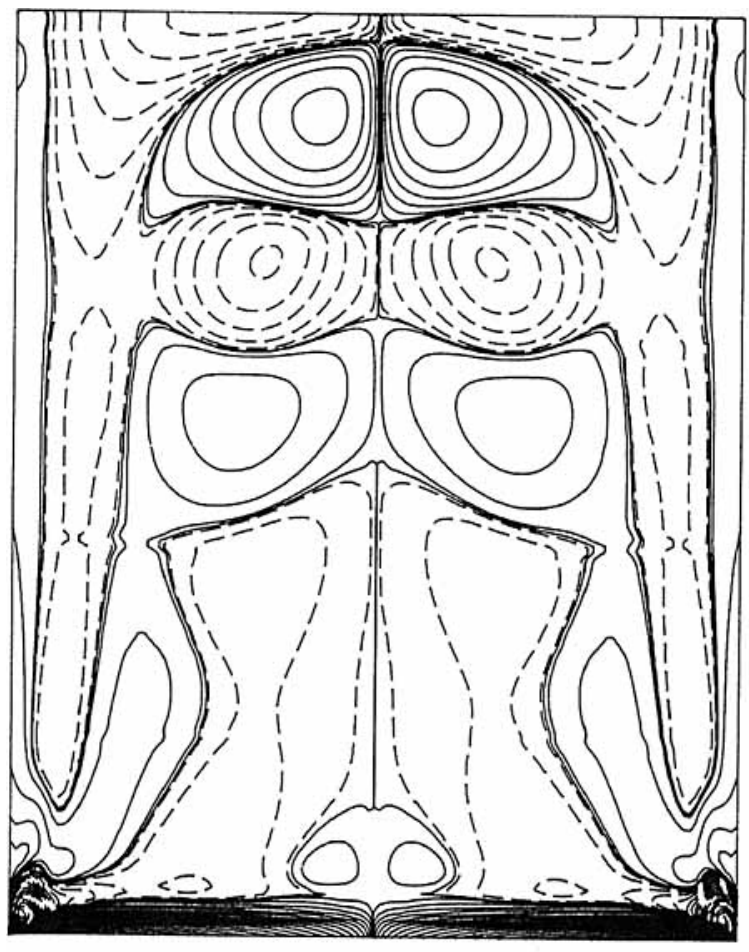

$\min .=0.19$

$\max .=0.33$

Figure 3. Contour plot of $v^{2} / r-(1 / \rho)(\partial p / \partial r)$ in the meridional plane for $H / R=2.5$ and $R e=1994$. The contour levels are non-uniformly space, with 20 positive and 20 negative levels determined by c-level $(i)=\operatorname{Max}($ variable $) \times(i / 20)^{3}$ and $\operatorname{c-level}(i)=\operatorname{Min}($ variable $) \times(i / 20)^{3} \quad$ respectively. The contours are plotted at $t=1000$, by which time a steady state has been reached.

head through the action of viscous stresses in the boundary layers near the surface of the volume, as noted in Part 1 . There is no reason why the radial distributions of $\Gamma$ and $\mathscr{H}$ should lead to $\eta=0$ (equation (3)), or to cyclostrophic balance (equation (1)). Quite the contrary, in fact, since the turning of the flow from radially inward towards the lower rotating endwall requires the centrifugal force to exceed the radial pressure gradient. This is supported by figure 3 which shows the calculated contours of $v^{2} / r-(1 / \rho)(\partial p / \partial r)$ for the case $R e=1994, H / R=2.5$. These contours provide an interesting view of the force field; they are not surprising in the sense that they accord with the waviness of the stream surface and the corresponding sinusoidal-like variation in the radial acceleration.

The reason for the initial 'overshoot' in the divergence of the stream surfaces and the subsequent waviness can be seen as follows. From (4), we have

$$
\frac{\partial u}{\partial z}=\frac{\Gamma}{r} \frac{\mathrm{d} \Gamma}{\mathrm{d} \psi}-r \frac{\mathrm{d} \mathscr{H}}{\mathrm{d} \psi}+\frac{\partial w}{\partial r} .
$$

For the confined swirling flow, if $z=z_{0}$ where $u=0$ and $\partial u / \partial z$ has a local extrema, i.e. where a stream surface has its smallest radius, then on a particular stream surface of radius $r=\sigma(z)$, whose radius is $\sigma=\sigma_{0}$ at $z=z_{0}$,

$$
\left.\frac{\partial u}{\partial z}\right|_{\sigma}-\left.\frac{\partial u}{\partial z}\right|_{\sigma_{0}}=A\left(\frac{1}{\sigma}-\frac{1}{\sigma_{0}}\right)-B\left(\sigma-\sigma_{0}\right)+\left.\frac{\partial w}{\partial r}\right|_{\sigma}-\left.\frac{\partial w}{\partial r}\right|_{\sigma_{0}}
$$


where $A$ and $B$ are the particular values of $\Gamma \mathrm{d} \Gamma / \mathrm{d} \psi$ and $\mathrm{d} \mathscr{H} / \mathrm{d} \psi$ on this stream surface.

An approximation for $\partial u /\left.\partial z\right|_{r}$ on the stream surface can be obtained by noting that

and

$$
\begin{gathered}
\left.\frac{\partial u}{\partial z}\right|_{r}=-\left.r u \frac{\partial u}{\partial \psi}\right|_{z}+\left.\frac{\partial u}{\partial z}\right|_{\psi}, \\
\left.\frac{\partial u}{\partial r}\right|_{z}=\left.r w \frac{\partial u}{\partial \psi}\right|_{z}
\end{gathered}
$$

from which it follows, since $u=w \sigma^{\prime}$ (equation $(6)$ ) on $\psi=$ constant, that

$$
\left.\frac{\partial u}{\partial z}\right|_{r}=w(z) \sigma^{\prime \prime}(z)+w^{\prime}(z) \sigma^{\prime}(z)-\left.\frac{u}{w} \frac{\partial u}{\partial r}\right|_{z}
$$

or in terms of a small perturbation linearization of the form $u=\epsilon u_{1}(r, z), w=$ $w_{0}+\epsilon w_{1}(r, z)$ and $\sigma=\sigma_{0}+\epsilon \sigma_{1}(z)$, to first order $\partial u / \partial z \approx w \sigma^{\prime \prime}$. Thus an approximation to (11) which allows some insight to be gained into the shape of the stream surface downstream from $z_{0}$ is

$$
w(\sigma) \sigma^{\prime \prime}-w(\sigma) \sigma^{\prime \prime}\left(z_{0}\right) \approx A\left(\frac{1}{\sigma}-\frac{1}{\sigma_{0}}\right)-B\left(\sigma-\sigma_{0}\right)+\left.\frac{\partial w}{\partial r}\right|_{\sigma}-\left.\frac{\partial w}{\partial r}\right|_{\sigma_{0}}
$$

Immediately downstream of $z_{0}$, the diverging stream surface implies an increasing $\sigma$ and $\sigma^{\prime \prime}$ is expected to decrease, i.e. $\sigma^{\prime \prime}(z)<\sigma^{\prime \prime}\left(z_{0}\right)$ because $A\left(1 / \sigma-1 / \sigma_{0}\right)$ and $-B\left(\sigma-\sigma_{0}\right)$ are both negative, assuming, in accord with $\$ 2.2$, that since breakdown occurs, $\alpha_{0}>\beta_{0}$ and $B$ is, therefore, positive. Note however that $\partial w /\left.\partial r\right|_{\sigma}-\partial w /\left.\partial r\right|_{\sigma_{0}}$ is expected to be positive because diverging stream surfaces imply by continuity a reduction in $w$, and a relatively larger reduction in $w$ is expected near $r=0$ than at large $r\left(\partial w /\left.\partial r\right|_{\sigma_{0}}\right.$ is expected to be negative). Thus, downstream of $z_{0}, \sigma^{\prime \prime}$ is expected to decrease, but the rate at which this occurs will depend on $w$. The continued divergence of the flow and corresponding reduction in $\sigma^{\prime \prime}$ therefore leads to a radius of the stream surface at which $\sigma^{\prime \prime}=0$ (infinite radius of curvature of the stream surface). At this point the local slope $\sigma^{\prime}$ is a maximum. Since the flow continues to diverge downstream from this point, $\sigma^{\prime \prime}$ becomes negative and the stream surface begins to reduce in slope, turning back towards the axis.

An alternative explanation for the flow divergence and subsequent turning of the flow towards the axis may be sought in terms of equation (1). An imbalance in $v^{2} / r-$ $(1 / \rho)(\partial p / \partial r)$ gives rise to a positive value of $\sigma^{\prime \prime}$. On this diverging stream surface, $v^{2} / r$ is decreased owing to the conservation of $\Gamma$ and, at the same time, $w$ and $v$ are reduced owing to the divergence, leading to an increased pressure with relatively larger changes for small $r$ and a reduction in the radial pressure gradient. While not self-evident, the reduction in $v^{2} / r$ must exceed the reduction in $\partial p / \partial r$ since, as outlined above, $\sigma^{\prime \prime}$ approaches zero.

The fact that the divergence 'overshoots' and that a wave in the stream surface results may be seen approximately by linearizing (12) about the radius $\sigma=\sigma_{1}$ at which $\sigma^{\prime \prime}=0$. Thus for $\sigma=\sigma_{1}+\xi(z)$, then from (12)

$$
w_{1} \xi^{\prime \prime} \approx-\left(\frac{A}{\sigma_{1}^{2}}+B\right) \xi
$$




$\begin{array}{cccc}R e & \lambda \text { (eq. (15)) } & \lambda \text { (eq. (14)) } & \lambda \text { (num. soln.) } \\ 1600 & 0.64 & 0.87 & 1.03 \\ 1800 & 0.55 & 0.81 & 0.82 \\ 1918 & 0.57 & 0.72 & 0.77 \\ 1942 & 0.70 & 0.72 & 0.77 \\ 1994 & 0.66 & 0.72 & 0.77 \\ 2126 & 0.61 & 0.69 & 0.75 \\ 2494 & 0.66 & 0.61 & 0.72\end{array}$

TABLE 1. Estimates of the wavelengths of the oscillations in the displacement of the stream surface determined (i) from the expressions given by (14) and (15) and (ii) from the periodicity in the contours of $v^{2} / r-(1 / \rho)(\partial p / \partial r)$ for various $R e$ as indicated and $H / R=2.5$

where perturbations in $\partial w / \partial r$ have been neglected compared with $\partial u / \partial z$. A wave in the stream surface is therefore established with wavenumber $k$ given by $w_{1} k^{2} \approx$ $A / \sigma_{1}^{2}+B$.

The shape of a characteristic stream surface in the core flow downstream of $z_{0}$ can be summarized as follows. The stream surface $\sigma(z)$ diverges from $\sigma_{0}$ to $\sigma_{1}$, and $\sigma^{\prime \prime}$ is reduced from a maximum to zero. At the radius $\sigma=\sigma_{1}$, the positive slope $\sigma^{\prime}$ is a maximum and the flow continues to diverge, $\xi>0$, but $\sigma^{\prime \prime}$, or $\xi^{\prime \prime}$, becomes negative and the surface begins to turn towards the axis. This overshoot of the radial displacement leads to a subsequent oscillation in the displacement of the stream surface about $\sigma=\sigma_{1}$, i.e. $\xi=0$. The wavelength of this displacement is approximately

$$
\lambda \approx 2 \pi w_{1}^{\frac{1}{2}}\left(\frac{\Gamma}{\sigma_{1}^{2}} \frac{\mathrm{d} \Gamma}{\mathrm{d} \psi}+\frac{\mathrm{d} \mathscr{H}}{\mathrm{d} \psi}\right)^{-\frac{1}{2}}
$$

where $w_{1}$ is the axial velocity at $r=\sigma_{1}$.

Using the expressions for $A$ and $B$ from $\$ 2.2$, the wavelength can also be approximated by

$$
\frac{\lambda}{\sigma_{1}} \approx 2 \pi\left(\frac{w_{1}}{\eta_{0} \sigma_{0}}\right)^{\frac{1}{2}}\left[\frac{\sigma_{1}^{2}}{\sigma_{0}^{2}}\left(\frac{\alpha_{0}}{\beta_{0}}-1\right)+\frac{\alpha_{0}}{\beta_{0}}\right]^{-\frac{1}{2}}
$$

A comparison between the wavelength evaluated from (15) and that determined from the periodicity in the numerical solution is presented in table 1 . The wavelength predicted by (15) was evaluated on a stream surface where $\Gamma$ and $\mathscr{H}$ are approximately constant (selected by comparing the contours of $\Gamma, \mathscr{H}$ and $\psi$ as in figures $2(f)$ and 3 from Part 1). The wavelength from the numerical solution was determined from the contours of $v^{2} / r-(1 / \rho)(\partial p / \partial r)$. Of course, the wavelength found in both cases can be regarded only as a typical wavelength. As expected the agreement (approximate) in table 1 between the inviscid prediction of (15) (and (14)) and the numerical solution increases with increasing Reynolds number. The difference between the predictions of (14) and (15) reflects the fact that (14) is evaluated in the region of the waves, where the flow is most inviscid, whereas (15) assumes inviscid flow from $z_{0}$ where the radius of the stream surface is a minimum. Closer examination of figures $2(f)$ and 3 from Part 1 shows that the approximation that $\Gamma$ and $\mathscr{H}$ are constant on stream surfaces is not accurate in this region. Particularly at lower Reynolds numbers it is not surprising therefore that the azimuthal viscous stresses should lead to some additional reduction in the azimuthal component of vorticity in this region and correspondingly that (9) would predict a 
larger radius than the numerical solution, for a given reduction in $\eta$. This is consistent with the prediction from (15) of a shorter wavelength than is found from the numerical solution.

As a check on whether the model describes the principal features of the flow, however, the agreement seems satisfactory. In accord with this discussion and that of $\$ 2.2$ the subsequent development of a second recirculation zone could be expected if the wave is of sufficient amplitude for the subsequent diverging stream surface to develop sufficient negative azimuthal vorticity to again bring the axial velocity to zero. The converging stream surface, by contrast, generates positive azimuthal vorticity which accelerates the axial flow.

The principal features and physical mechanisms of the recirculation bubbles in the confined swirling flow are therefore comprehensible in terms of inviscid phenomena. The initial divergence from a narrow core is the result of the upstream radial distribution of $\mathscr{H}$ and $\Gamma$, the subsequent waviness of the stream surface is a result of a stationary inertial or centrifugal wave and the development of one or more recirculation zones is a consequence of the generation of negative azimuthal vorticity through the stretching and tilting of vortex lines in a diverging, swirling inviscid flow. At a Reynolds number above that at which a recirculation bubble first occurs, the apparent 'critical' dependence of the flow on $R e$, discussed in Part 1, arises essentially from the dependence of the radial distributions of $\Gamma$ and $\mathscr{H}$ (in the core flow) on the action of viscous stresses in the boundary-layer regions near the walls. At higher Reynolds number the core flow behaves in a broadly inviscid manner and the recirculation zones appear to have relatively little dynamical significance, except insofar as they are within the region of strong negative azimuthal vorticity.

These mechanisms, which appear to account for the principal features of the recirculation bubbles in the confined flow, led to our considering their applicability to swirling flows in pipes.

\section{Swirling flows in pipes and in a free stream}

\subsection{Preliminary considerations}

The relatively sudden appearance of a rapid divergence in the stream surfaces, and the corresponding appearance of recirculation zones, occurs in some swirling pipe flows and in the vortex breakdown observed in flows over delta wings at high angle of attack. A comparison with the 'breakdown' observed in the confined swirling flow suggests that these breakdown regions are qualitatively similar, at least up to the first recirculation region. An essential difference, however, is that for swirling pipe flows and to some extent the delta-wing flow, the 'upstream' region may be regarded as essentially a cylindrical flow for which initially $v^{2} / r=(1 / \rho)(\partial p / \partial r)$ or, in terms of (4)

$$
\frac{\partial u}{\partial z}=\frac{\Gamma \mathrm{d} \Gamma}{r} \frac{\mathrm{d} \mathscr{H}}{\mathrm{d} \psi}+\frac{\partial w}{\partial r}=0 .
$$

For this case, the question that is immediately posed is how does 'breakdown' begin ?

There has been a large number of numerical studies of this swirling pipe flow. One of the earliest examples is that of Kopecky \& Torrance (1973), where the timedependent axisymmetric Navier-Stokes equations were solved using an explicit finite-difference technique. The coarseness of their grid (typically, they used $11 \times 21$ grid points uniformally spaced) required them to consider low-Reynolds-number 


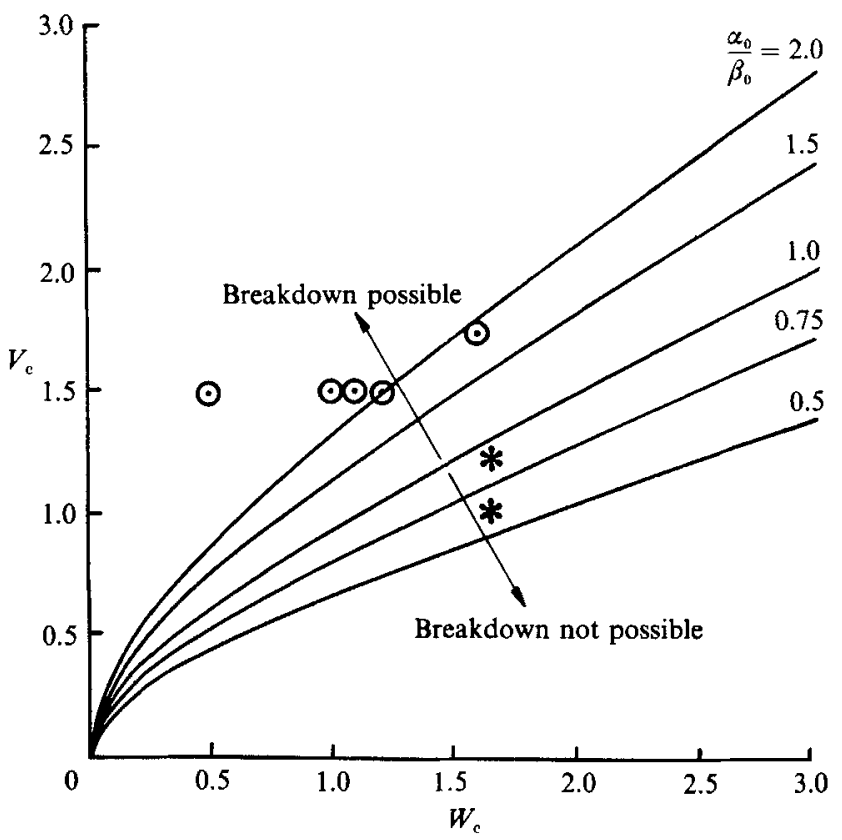

Figure 4. The dependence of the ratio of the velocity and vorticity helix angles $\alpha_{0} / \beta_{0}$ on the dimensionless azimuthal velocity $V_{\mathrm{e}}$ and axial velocity excess $W_{\mathrm{e}}$ at $r=a$ at the upstream boundary of a swirling pipe flow with azimuthal and axial velocity components given by (16) and (17) respectively. The circles indicate the location in $\left(V_{c}, W_{c}\right)$-space where the numerical solutions showed breakdown to occur and the stars indicate where breakdown was not found.

flows; it may also have been responsible for the solutions being steady and for the overly large axial extent, compared with the present study, of the recirculation zone. Grabowski \& Berger (1976), utilizing a much finer grid, were able to find solutions with a closed recirculation zone of similar spatial dimensions to those found experimentally by Sarpkaya $(\mathbf{1 9 7 1 b})$. Their results did not show the detailed structure of the bubble as found by Faler \& Leibovich (1978) in which a counterrotating vortex ring was measured inside the breakdown bubble. Also, Grabowski \& Berger (1976) solved only the time-independent Navier-Stokes equations. More recently, Krause, Shi \& Hartwich (1983) and Shi (1985) solved the time-dependent Navier-Stokes equations for swirling pipe flow. When the flow underwent vortex breakdown, they found that it did not reach a steady state and that the upstream boundary condition had a significant influence on the solutions at late times. These studies were the first to numerically reproduce the 'two-celled' internal structure of the breakdown bubble, first observed by Faler \& Leibovich (1978).

One of us (Lopez) has obtained time-dependent numerical solutions of the Navier-Stokes equations for various swirling flows in a pipe by the methods outlined in Part 1. The purpose of presenting these computations here is to provide a further test for the ideas put forward in $\$ 2$ and to demonstrate their more general applicability.

The particular family of flows that has been calculated is given at $t=0$ by a zero radial component of velocity, an azimuthal component of velocity

$$
v=\frac{a v_{\mathrm{c}}}{r\left(1-\mathrm{e}^{-1}\right)}\left(1-\mathrm{e}^{\left.-r^{2} / a^{2}\right)}\right.
$$


(a)

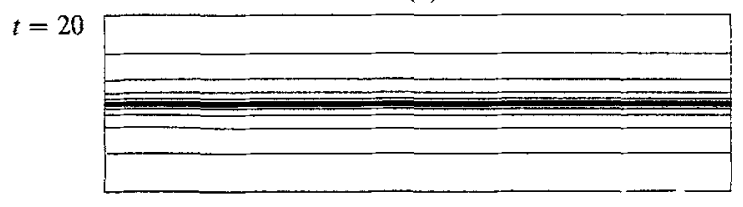

40

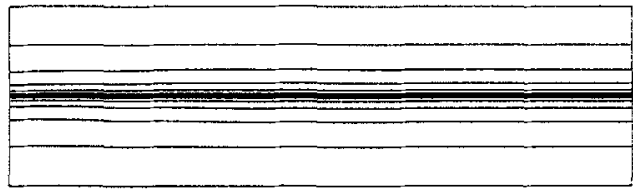

60

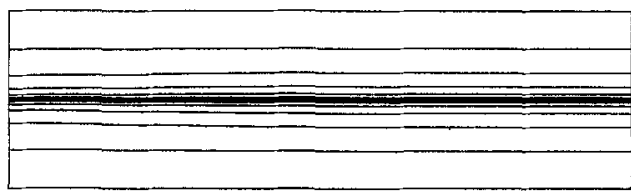

80

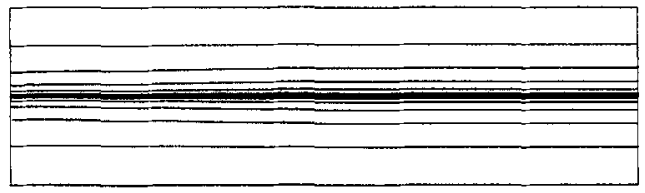

140

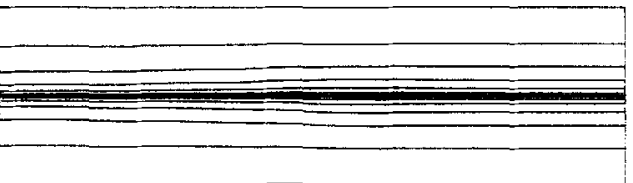

240
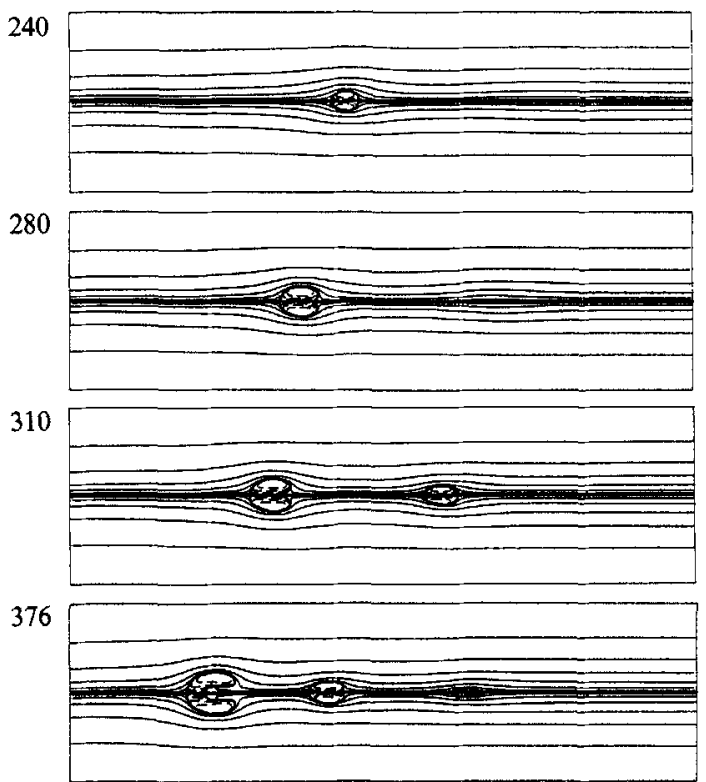

416

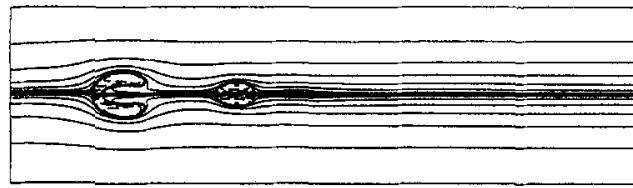

Figure 5(a). For caption see page 567. 
(b)

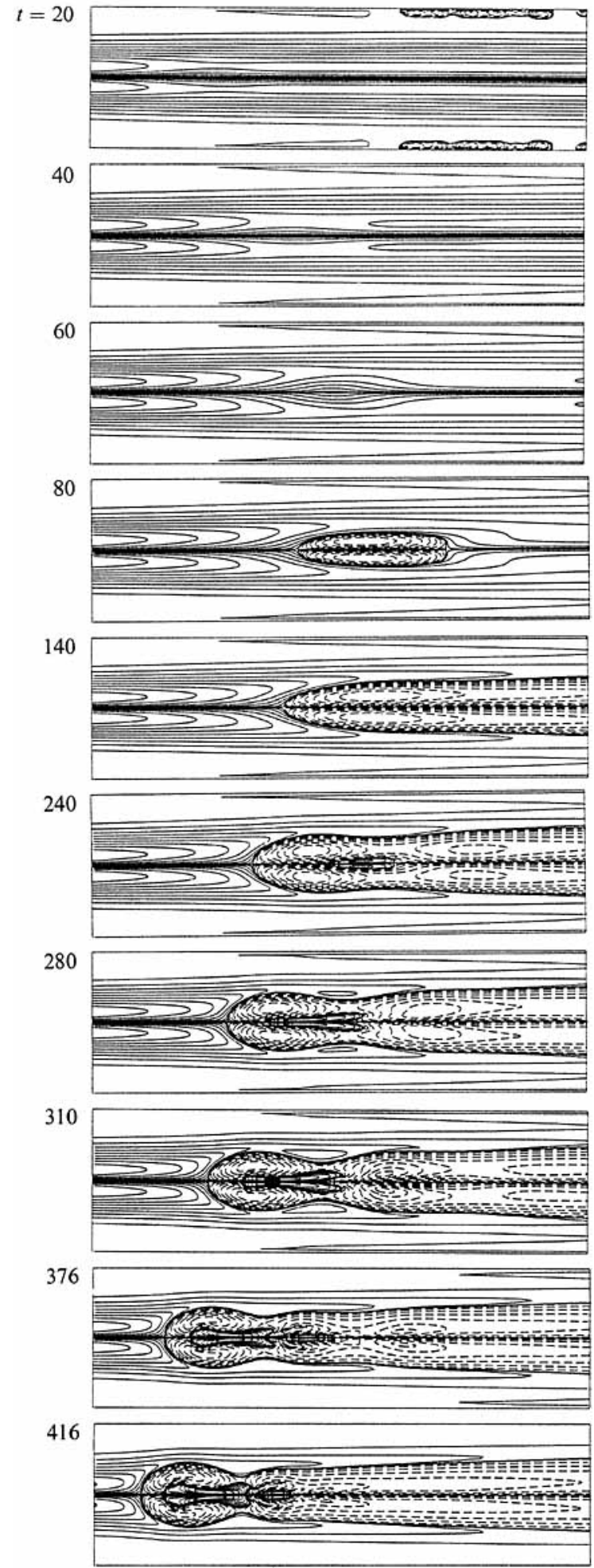

Fraure 5(b). For caption see page 567 . 
(c)

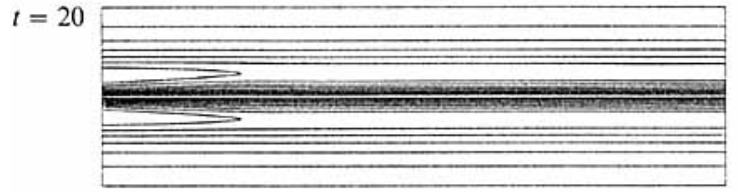

40

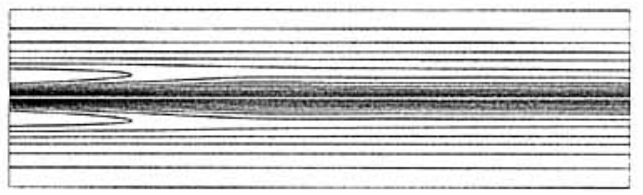

60

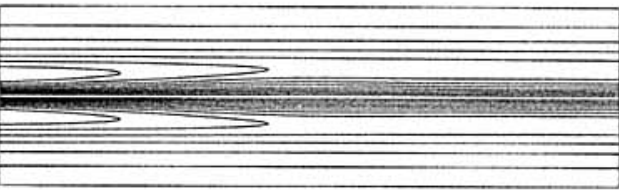

80

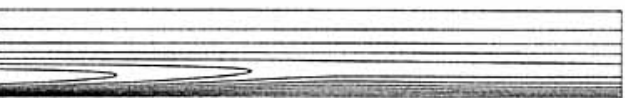

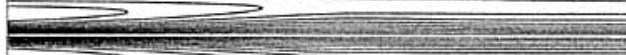

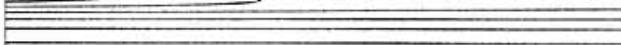

140

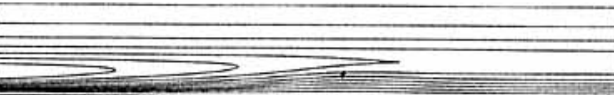
$\stackrel{\longrightarrow}{\longrightarrow}$

240

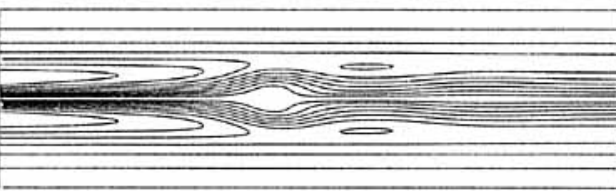

280

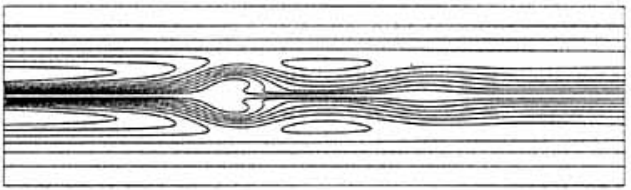

310

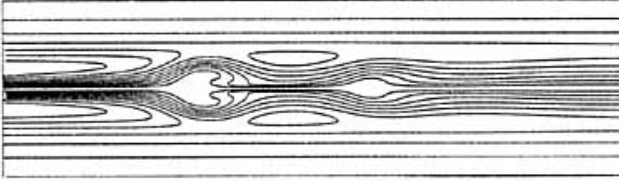

376

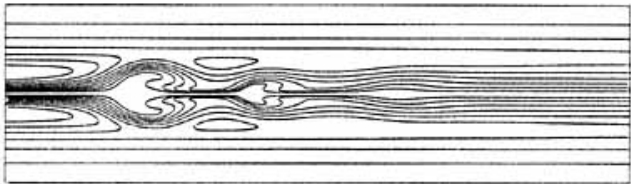

416

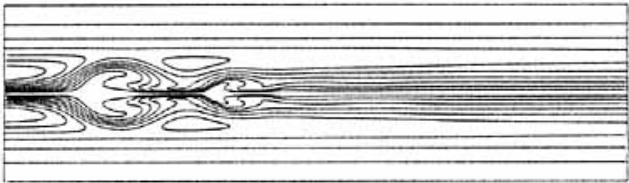

Figure $5(c)$. For caption see facing page. 
and an axial component of velocity

$$
w=W_{\infty}\left(1+W_{\mathrm{c}} \mathrm{e}^{\left.-r^{2} / a^{2}\right)} .\right.
$$

These distributions have been found to be good fits to various experimental observations of vortices undergoing breakdown (e.g. Garg \& Leibovich 1979). This azimuthal velocity distribution has the further merit that it has the same form as the exact solution for the diffusion of vorticity from a line vortex (Burgers 1940).

The distributions of velocity in (16) and (17) have the corresponding azimuthal and axial components of vorticity,

$$
\begin{gathered}
\eta=\frac{2 W_{\infty} W_{\mathrm{c}} r}{a^{2}} \mathrm{e}^{-r^{2} / a^{2}}, \\
\zeta=\frac{2 v_{\mathrm{c}}}{a\left(1-\mathrm{e}^{-1}\right)} \mathrm{e}^{-r^{2} / a^{2}},
\end{gathered}
$$

and

and in terms of the helix angles for velocity and vorticity discussed in $\S 2.2$, on stream surfaces at $z=0$

$$
\frac{\alpha_{0}}{\beta_{0}}=\left(\frac{\mathrm{e}}{\mathrm{e}-1}\right)^{2} \frac{V_{\mathrm{c}}^{2}}{W_{\mathrm{c}}} \frac{a^{2}}{r^{2}} \frac{\left(1-\mathrm{e}^{-r^{2} / a^{2}}\right)}{\left(1+W_{\mathrm{c}} \mathrm{e}^{-r^{2} / a^{2}}\right)}
$$

where $V_{\mathrm{c}}=v_{\mathrm{c}} / W_{\infty}$. Within the range $1<W_{\mathrm{c}}<2, \alpha / \beta$ does not vary substantially for stream surfaces for which $0<r / a<1$, and at $r / a=1$

$$
\frac{\alpha_{0}}{\beta_{0}}=\frac{W_{\mathrm{c}} \mathrm{e}^{2}}{(\mathrm{e}-1)\left(W_{\mathrm{c}}+\mathrm{e}\right)} \frac{V_{\mathrm{c}}^{2}}{W_{\mathrm{c}}^{2}} .
$$

This relationship between $\alpha_{0}$ and $\beta_{0}$ at $r / a=1$ for various values of $V_{\mathrm{c}}$ and $W_{\mathrm{c}}$ is shown in figure 4 and the region where $\alpha_{0}>\beta_{0}$ is marked. The particular values of $V_{\mathrm{c}}$ and $W_{\mathrm{c}}$ where the numerical solutions obtained led in time to the formation of recirculation bubbles are also given in this figure, along with two further cases $\left(\alpha_{0}=\right.$ $0.90 \beta_{0}$ and $\alpha_{0}=0.62 \beta_{0}, R e=300$ ) in which recirculation bubbles were not found after an integration time of 350 . (In both these cases an asymptotically steady state was reached.) For a particular flow to be discussed, $V_{\mathrm{c}}=1.5, W_{\mathrm{c}}=1.25$ and at $r=a, \alpha_{0} / \beta_{0}$ is $\mathbf{1} .95$.

The initial conditions for the calculated flows are that the velocities at $t=0$ for all $z$ are given by (16) and (17) and the upstream boundary condition is that these velocities are imposed for $t>0$. The radial boundary condition at $r=R$ is $u=0$, $\partial v / \partial r=0$ and $\eta=0$. The usual outflow condition, $\partial / \partial z=0$ is applied. (A further discussion of these conditions and their implementation is in the Appendix.) With these initial and boundary conditions the presence of viscosity is essential for the evolution of this flow - inviscid flow would not change with time. An example of the development of the flow for a particular Reynolds number is shown in figure 5. Of course, at very low Reynolds number, viscous diffusion dominates the flow

Figure 5. The evolution of the axisymmetric vortex breakdown phenomenon in swirling pipe flow for the case of $V_{\mathrm{c}}=1.5, W_{\mathrm{c}}=1.25$ and $R e=W_{\infty} a / \nu=250$. The computational domain has a radial extent of $5 a$ and an axial extent of $35 a$ in which 351 grid points are uniformly distributed in the axial direction and $\mathbf{5 1}$ grid points are non-uniformly distributed in the radial direction and are concentrated near $r=0$. The time increment for the evolution is $\delta t=0.01$ and the non-dimensional times at which the instantaneous contours of $(a) \psi,(b) \eta$ and $(c) v$ are indicated in each figure. 
development. At higher Reynolds numbers, however, we have found that for the same initial cylindrical velocity distributions, the time taken before a recirculation zone appears increases with Reynolds number (defined as $R e=W_{\infty} a / \nu$ ). Unlike the confined swirling flow, for these flows it is the diffusion of axial vorticity which leads initially to $v^{2} / r-(1 / \rho)(\partial p / \partial r)$ becoming positive. Alternatively, we anticipate that it must be the diffusion of vorticity that leads to a radial redistribution of $\Gamma$ and to the stretching and tilting of vortex lines (due to the axial change in $v$ ), with a corresponding reduction in the initial positive azimuthal component of vorticity with axial distance, and the subsequent beginning of an 'inviscid' breakdown process.

This broad view of the likely role of viscosity and the development of azimuthal vorticity is illustrated in the calculations of $\psi, \eta$ and $v$ shown in figure 5 and leads to the more detailed considerations of $\$ 3.2$.

In passing, we note that none of the cases that led to a recirculation bubble ever settled down to a steady state. The bubble would migrate upstream until halted by the influence of the time-independent upstream boundary conditions.

\subsection{Physical mechanisms}

In considering the results shown in figure 5, particular attention is drawn to the reduction of the azimuthal velocity and vorticity with distance downstream. Initially, this reduction is due to viscous effects, but it is clear that by $t=40$ there is a slight divergence of streamlines which, by the inviscid mechanisms of $\S 2$, contributes to this reduction. At $t=\mathbf{8 0}$ this azimuthal vorticity has been reduced to zero on stream surfaces at a downstream distance of about $20 \mathrm{a}$ and out to a radius of about $a$ and a further divergence of these stream surfaces has generated a negative azimuthal component. The inviscid mechanism, described in the discussion of equation (9), might be said to drive this further development of a negative azimuthal component leading, by $t=240$, to a small recirculation zone on the axis, rapid changes in the azimuthal vorticity ahead of this region where the streamlines diverge, and the evident propagation upstream of the region of negative azimuthal vorticity due to its own induced velocity. The subsequent appearance of a second recirculation zone located at a distance downstream where the outer streamlines diverge and where the azimuthal component of the vorticity has a second maximum is consistent with the establishment of a wave on the outer stream surfaces by essentially the same mechanism as in the confined swirling flows.

Further insight into the mechanics of the flow is obtained from a detailed comparison between the results shown in figures $6(b), 6(c)$ and $6(d)$. Figures $6(a)$ and $6(b)$ show the development of the flow for which $V_{c}=1.75, W_{\mathrm{c}}=1.6$ and $R e=300$ (with a corresponding $\alpha_{0} / \beta_{0}=1.91$ ) from $t=227$ to $t=250$. Figure $6(c)$ shows a corresponding development in an unphysical case in which, for the above flow, at $t=227$ the viscosity is suddenly doubled, (i.e. Reynolds number reduced from 300 to 150 ). Figure $6(d)$ is a case in which the viscosity is suddenly halved ( $R e=600$ ) at $t=227$. A comparison between figures $6(b)$ and $6(c)$ shows that the subsequent effect of a sudden increase in the viscosity is to diffuse the axial vorticity and increase the initial divergence of streamlines but reduce the magnitude of the maximum negative component of azimuthal vorticity from -1.84 to -1.37 , to reduce the size of the recirculation bubble. By contrast, comparison between figures $6(b)$ and $6(d)$ shows that reducing viscosity reduces the diffusion of axial vorticity which reduces the upstream divergence of streamlines; the presence of the bubble and divergence of the streamlines immediately ahead of it and the reduced diffusion of axial vorticity increases the magnitude of the maximum negative component of azimuthal vorticity 


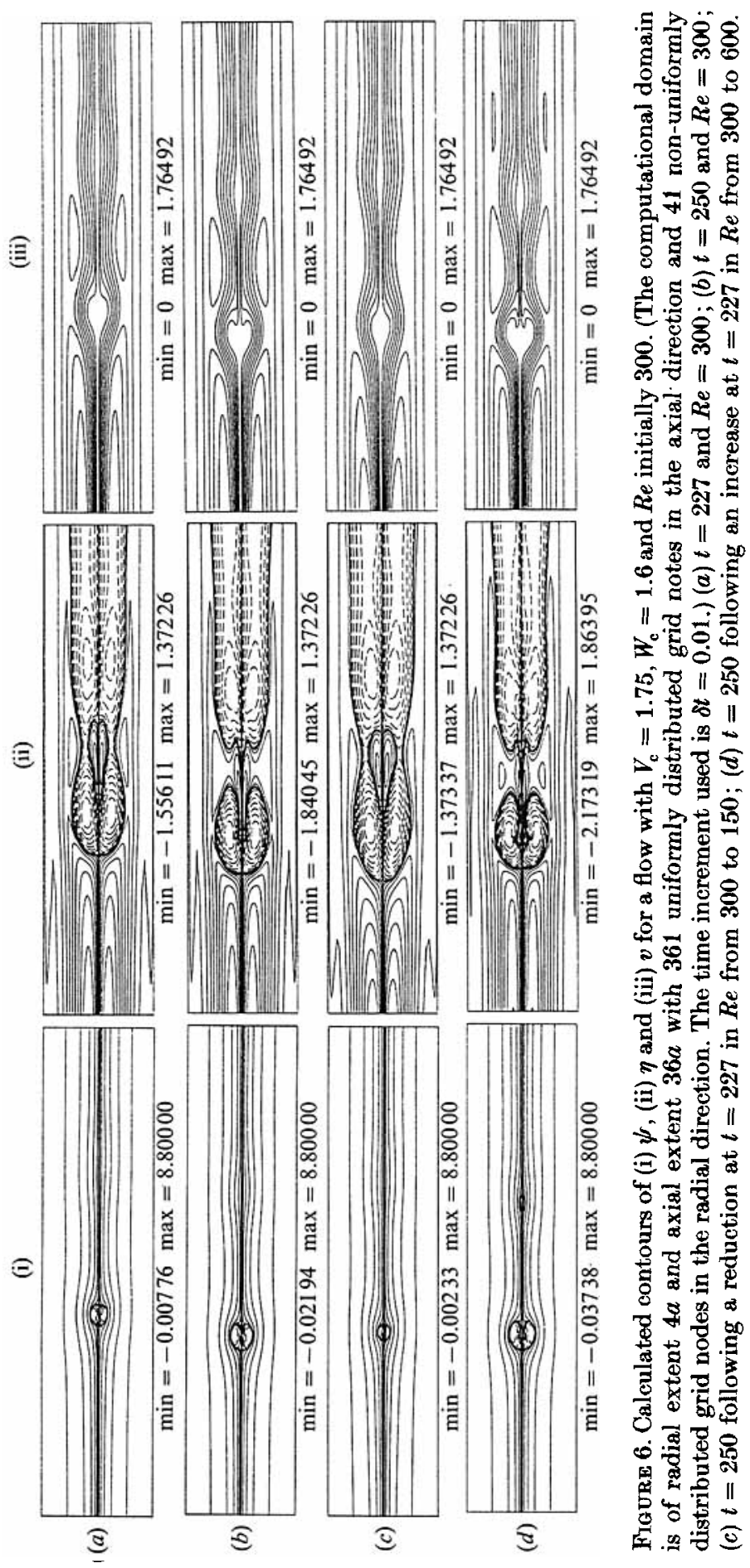




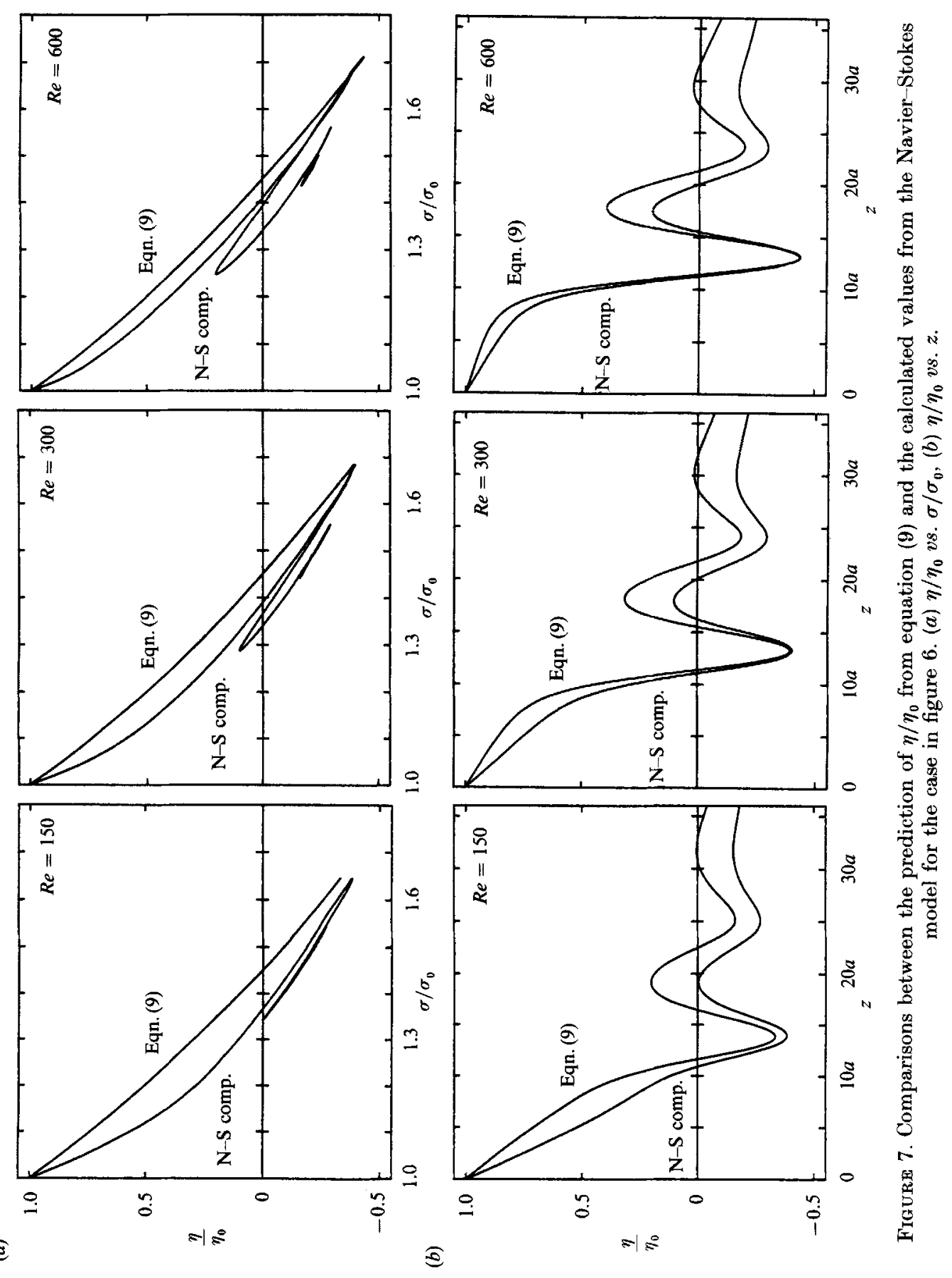


that is reached from -1.84 to -2.17 . The bubble is correspondingly larger, the amplitude of the wave on the outer stream surface is greater and a small second recirculation zone begins to emerge.

These results are quite consistent with the discussion in $\$ 2.2$. For this flow the necessary criterion for breakdown to occur $\left(\alpha_{0}>\beta_{0}\right)$ is satisfied. The development of the flow requires viscosity to initiate a reduction of the azimuthal components of vorticity and velocity with distance downstream and to initiate the divergence of the streamlines. However, the development of the breakdown results essentially from the inviscid process described in $\$ 2.2$. The reduction in viscosity introduced after a bubble has appeared, figure $6(d)$, shows the dominant role that this inviscid mechanism plays. In some respects the phenomenon in this swirling pipe flow case is not unlike boundary-layer transition in the general sense that the initial development (Tollmien-Schlichting waves in boundary-layer transition) depends on viscosity and the final transition process is a consequence of 'positive feedback' in the generation, in that case, of a longitudinal component of vorticity.

The dominant role played by the inviscid mechanism can be demonstrated further by comparing directly the calculated azimuthal vorticity on a stream surface with the prediction obtain from (9). This comparison was made by selecting a stream surface $\psi_{1}$ in the numerical solution at a given time and choosing an upstream station $z_{0}$, where the flow is essentially cylindrical. For the examples used here, $z_{0}=0$ and $\psi_{1}$ has radius $\sigma_{0}=a$ at $z_{0}$. From the numerical solutions, the radius $\sigma$, and the azimuthal vorticity $\eta$, are determined at various axial stations $z_{i}$. These values of $\eta$ are then compared with those predicted from (9) for the $\sigma\left(z_{i}\right)$ values of the numerical solutions. The values of the helix angles of velocity and vorticity $\alpha_{0}$ and $\beta_{0}$ at $z_{0}$ are found from (18).

Figure 7 corresponds to the cases in figures $6(b), 6(c)$ and $6(d)$ and shows $\eta / \eta_{0}$ from the numerical calculations and from (9) plotted against $(a) \sigma / \sigma_{0}$ and $(b) z$. All curves shown in figure $7(b)$ show the rapid development of negative $\eta$ in the vicinity of the recirculation bubbles. The agreement is best in this region. This might be expected since, in the viscous calculations, the change in $\sigma$ well upstream of the recirculation region is primarily due to viscous diffusion, whereas in the recirculation region the inviscid mechanism is dominant. The figure shows that as $R e$ is increased, $\partial \eta / \partial z$ upstream of the recirculation zone tends towards zero and the rapid reduction in $\eta$ near the recirculation zone is predicted increasingly accurately by (9). As expected, the overall agreement between the numerical Navier-Stokes calculations and the inviscid theory increases as $R e$ is increased.

\subsection{Comparison with experiment}

While more detailed comparisons with experiment over a wide range of parameters have not yet been made, the essential features of the evolution of an axisymmetric vortex breakdown are evident. The description by Sarpkaya (1971a) of the initial axisymmetric swelling of the vortex core together with Escudier's (1986) evolutionary sequence in his figure 9.12 (reproduced here in figure 8 ) are seen in the calculation of the axisymmetric evolution of figure 5. Noting that in the calculations the timescale of the evolution is given by $a / W_{\infty}$, and recalling that the Reynolds number is based on the core radius $a$ rather than the pipe diameter, we find that the timescale of the evolution compares favourably (at a comparable $R e$ ) with that obtained in Escudier's (1986) experiment. (Owing to the lack of a quantitative measurement in the experiment, only a rough estimate of the core diameter $a$ could be made.) A comparisons between the structure of the leading bubble with the flow 
(a)

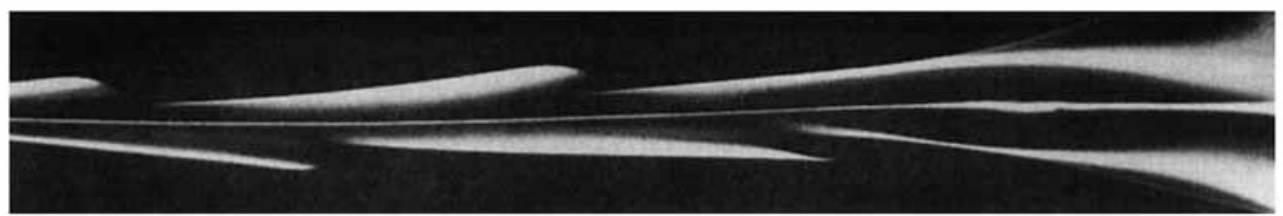

(b)

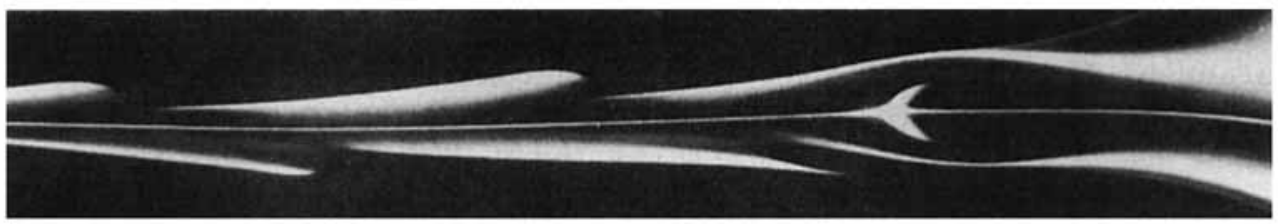

(c)

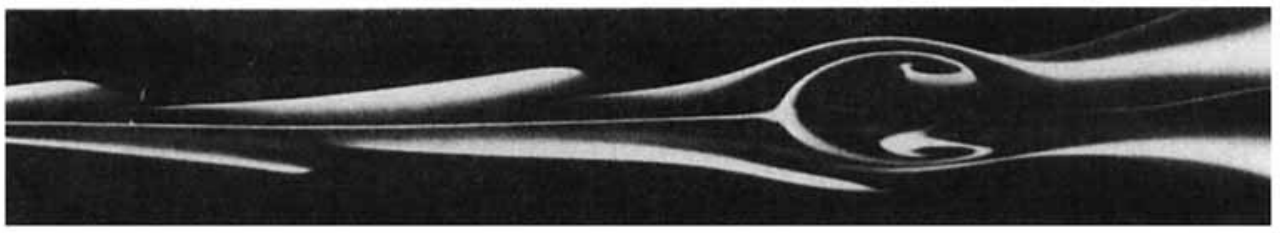

(d)

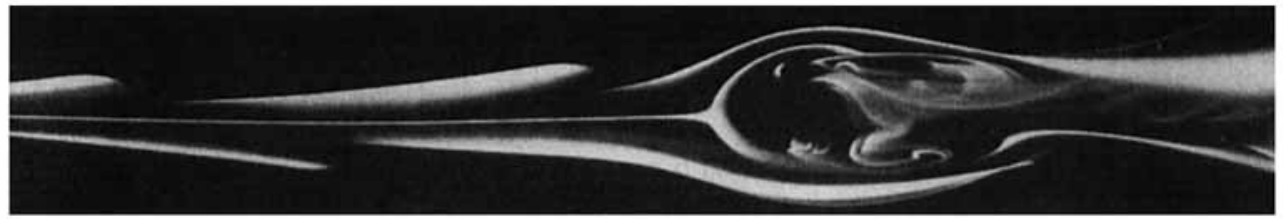

$(e)$

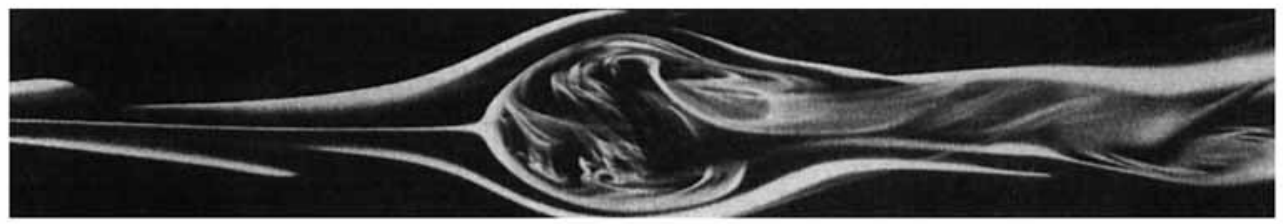

$(f)$

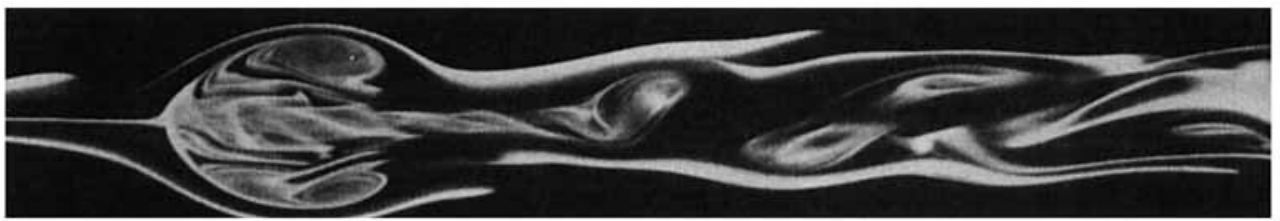

Figure 8. Escudier's (1986) observation of the formation and propagation of an axisymmetric (bubble type) vortex breakdown downstream of a guide-vane system, (vane angle $\phi=70^{\circ}$ and the Reynolds number, based on the pipe diameter and mean axial velocity, is 960 .) (a) $t=0,(b) 4 \mathrm{~s}(c)$ $8 \mathrm{~s},(d) 12 \mathrm{~s},(e) 20 \mathrm{~s},(f) 52 \mathrm{~s}$.

visualizations of Escudier (1986) shows good agreement. For comparable times in both the experiment and the calculation, the bubble migrates upstream approximately the same distance (four bubble diameters) from the point of initial swelling of the core. The appearance of a second breakdown zone at about one bubble diameter downstream from the leading bubble is evident in both experiment and calculation. In the calculation this second recirculation zone is very unsteady by comparison with the leading bubble and the flow visualization is suggestive of asymmetries in this region of the flow, whereas the leading bubble is evidently largely axisymmetric. The asymmetries of the second bubble may be an artifact of the form 
noted by Neitzel (1988) from the visualization of unsteady axisymmetric swirling flows. Also clearly evident in the computed leading bubble is the 'two-celled' structure first identified in bubble breakdowns by Faler \& Leibovich (1978). The main outer cell, or vortex ring, has an associated negative azimuthal component of vorticity and the weaker inner ring has a positive azimuthal component of vorticity.

\section{Concluding remarks}

Many of the features of vortex breakdown have been well recognized previously. The early works of Squire (1960) and Benjamin $(1962,1967)$ have been the starting point for many subsequent studies and Hall (1972) has summarized the contributions and shortcomings of various 'explanations' for vortex breakdown that have been proposed. For the confined flow and the swirling pipe flow, however, we have not found the 'conjugate flow' theory or the idea of a 'critical state' of particular assistance in illuminating the mechanics of these flows. The conjugate flow theory assumes a cylindrical flow upstream, whereas in the confined flow there is no region far upstream of breakdown in which the centrifugal forces balance the radial pressure gradient. Similarly, there is no evidence of the importance of energy dissipation in the outer flow. In the swirling pipe flow case, the calculations and physical mechanisms for the generation of negative azimuthal vorticity that we have considered depend initially on the diffusion of axial vorticity, whereas the conjugate flow theory assumes an inviscid development. It seems nevertheless true that, having established breakdown, the azimuthal vorticity that is generated propagates upstream on an inertial timescale and the diffusion of vorticity, which is initially responsible for the breakdown, is of lesser significance and continues to operate on the much slower viscous timescale. Also we have not found evidence of a disturbance downstream that propagates upstream and is responsible for breakdown.

From the discussion in this paper, the essential feature of vortex breakdown is the generation of negative azimuthal vorticity. For the confined flow, the rapid generation of this component of vorticity is a consequence of the upstream distribution of $\Gamma(r)$ and $\mathscr{H}(r)$ and the non-cylindrical nature of the upstream flow. For the swirling pipe flow, we find the initial reduction in the azimuthal component of vorticity to be due to viscous diffusion and the resultant stretching and tilting of axial vorticity. As the flow begins to diverge, however, the further production of negative azimuthal vorticity is dominated by inviscid mechanisms and there is a close similarity between the confined flow and the swirling pipe flow in this region.

A preliminary application of these ideas to the more complex problem of vortex breakdown in delta-wing flows suggests that the radial and axial velocity distributions in the core flow which establish a characteristic $\alpha_{0} / \beta_{0}$ for each angle of attack will play a critical role in determining whether breakdown can occur and if so the strength and location of it. Similarly, by broad analogy with the swirling pipe flow, turbulent diffusion could be expected to be important if the flow initially is in approximate cyclostrophic balance. In this case increased turbulent diffusion would be expected to reduce the size of the recirculation bubble and to reduce the distance to breakdown. Of course, an outer flow which oreates diverging stream surfaces will shorten the distance to breakdown and increase the relative importance of the inviscid mechanism in reducing an initially positive helix angle of the vorticity. This effect of a diverging outer flow has been observed in swirling pipe flows and may be seen in a comparison between the diverging-pipe results of Sarpkaya (1971 $a$ ) and the constant-diameter results of Harvey (1962). 
The essential ideas that link the generation of negative azimuthal vorticity with the helix angles of the velocity and vorticity vectors will also be applicable to swirling flows past axisymmetric bodies located on the swirl axis. The inviscid mechanism from (9) could account, for example, for the sensitivity of these flows to the intrusion of external probes leading in some cases to a rapid development of vortex breakdown. For $\alpha_{0}>\beta_{0}$ the divergence of stream surfaces over a body could be expected to lead to a forward recirculation zone ahead of the body if $\alpha_{0} / \beta_{0}$ is sufficiently large or the body has a large enough diameter. Such a flow with a forward recirculation zone has been observed recently by $\mathrm{P}$. N. Joubert (private communication). As discussed in $\$ 2.2$, the flow for the case where $\beta_{0}>2 \alpha_{0}$ is much less clear; the negative feedback that would accompany an increase in radius of a stream surface in this case suggests that some dissipative process ahead of the body will occur.

\section{Appendix}

The main difficulty in the numerical solution of the vortex breakdown problem for swirling pipe flow lies in the specification of the boundary conditions. The boundary condition on the axis of symmetry is obvious but the other three boundary conditions require a higher level of idealization.

The upstream boundary condition at $z=0$ is the one of main concern. Owing to the limited number of available grid nodes $(\mathrm{a} 101 \times 501$ grid has been an upper limit in this calculation) the swirl generator, i.e. guide vanes, and the pipe inlet could not be simulated at the same time as the flow inside the pipe 'test-section'. Hence, the upstream boundary must be placed at some location downstream of the inlet and upstream of the 'test-section'. This requires an assumption that the flow at the upstream boundary is locally cylindrical and independent of time throughout the evolution of the calculated flow. Over the past decade, there have been numerous laser Doppler anemometer measurements of the azimuthal and axial components of the velocity (e.g. Faler \& Leibovich 1978; Escudier, Bornstein \& Zehnder 1980; Uchida, Nakamura \& Ohsawa 1985) and it has been found that the expressions (16) and (17) provide a good fit to the experimental distributions well upstream of the location where vortex breakdown occurs. For those numerical cases where vortex breakdown first appears well downstream of the inlet it would seem therefore that these expressions, used as boundary conditions, are a reasonable approximation.

In those cases where the breakdown bubble migrates upstream a change in the velocities near the upstream boundary as a result of this migration is a clear indication that the numerical flow has become 'unphysical'. In many cases there is a substantial evolution of the 'breakdown' before this occurs. The weakness in this boundary condition requiring further evaluation, however, is that the viscosity changes discontinuously at the boundary and correspondingly gradients in $v$ (and therefore $u$ ) change relatively rapidly at low Reynolds number, near the boundary.

Of lesser importance, but with its own problems, is the specification of the radial and downstream boundary conditions. At the downstream boundary, the usual uniform outflow condition is specified. In the numerical experiments performed, so long as no rotational flow enters into the computational domain through this boundary, changes in the location of this boundary demonstrated that the effect of the downstream boundary was negligible in these calculations, in the development of the initial breakdown. However, under some conditions, it was found that multiple recirculation bubbles formed (much like those reported by Sarpkaya 1971 $a$ ) and 
when a bubble forms very near the downstream boundary (which often happens when the initial azimuthal component of vorticity is large and positive as a result of a strong axial jet), then the assumption of uniform outflow is clearly violated and the calculation becomes unphysical. A crude remedy used was to extend the computational domain further downstream.

The radial boundary condition is a somewhat less difficult problem. Typically, it was found that if the radial boundary is placed at a radius of at least two core diameters, then the numerical evolution is weakly affected by this boundary. A similar weak influence of the radial boundary was also found in the experiments of Harvey (1962). For the solutions presented, the boundary is at five vortex core radii from the axis. In the model, the computational domain is stretched in the radial direction so that there is a concentration of grid nodes near $r=0$ thus allowing proper resolution of the dynamies in the core region. The radial boundary condition is taken to simulate a limiting streamline, in the irrotational region of the flow, which remains parallel to the pipe wall. This is physically reasonable so long as the diffusion of vorticity is not large enough to invalidate the irrotational approximation and the boundary layer on the pipe wall (which is not computed) remains thin and attached.

An interesting and relevant observation to be made is the difference between the 'downstream' boundary condition in the case of confined swirling flow and that in other flows in which vortex breakdown occurs. In these others cases (e.g. vortical flow over delta-winged aircraft, swirling pipe flows, Ward-type cyclone chambers, tornados and waterspouts) the downstream boundary condition on the vortex core is 'free', whereas in the confined swirling flow the rotating endwall forms the downstream boundary, which results in the physical boundary condition that $v=0$ at $r=0$. The vortex core is therefore fixed to the centre of the downstream boundary. Having this fixed downstream boundary condition on the vortex core perhaps accounts for the fact that the 'spiral' form of vortex breakdown which is evident in the other examples of vortex breakdown is not evident at the values of $\Omega R^{2} / \nu$ and $H / R$ reported in the confined flow experiments.

\section{REFERENCES}

Benjamin, T. B. 1962 Theory of the vortex breakdown phenomenon. J. Fluid Mech. 14, 593-629.

Benjamin, T. B. 1967 Some developments in the theory of vortex breakdown. J. Fluid Mech. 28, 65-84.

Brown, G. L. \& Lopez, J.M. 1988 Axisymmetric vortex breakdown. Part II: Physical mechanisms. ARL Aero. Rep. 174 AR-004-573.

BURGERs, J. M. 1940 Application of a model system to illustrate some points of the statistical theory of free turbulence. Proc. Kon. (Ned.) Akad. v. Wetensch. Amsterdam XIIII, 2-12.

Escudier, M. P. 1986 Vortex breakdown in technology and nature. Von Karman Institute for Fluid Dynamics Lecture Series Programme. 10. Introduction to Vortex Dynamics, May 1986, Lecture 9.

Escudier, M. P., Bornstein, J. \& Z ZHNDER, N. 1980 Observations and LDA measurements of confined turbulent vortex flow. J. Fluid Mech. 98, 49-63.

Faler, J. H. \& Leibovich, S. 1978 An experimental map of the internal structure of a vortex breakdown. J. Fluid Mech. 86, 313-335.

Garg, A. K. \& Leibovich, S. 1979 Spectral characteristics of vortex breakdown flowfields. Phys. Fluids 22, 2053-2064.

Grabowski, W. J. \& Berger, S. A. 1976 Solutions of the Navier-Stokes equations for vortex breakdown. J. Fluid Mech. 75, 525-544.

Halt, M. G. 1972 Vortex breakdown. Ann. Rev. Fluid Mech. 4, 195-218.

HARVEY, J. K. 1962 Some observations of the vortex breakdown phenomenon. J. Fluid Mech. 14, $585-592$. 
Keller, J. J., EGLI, W. \& ExLEY, J. 1985 Force- and loss-free transitions between flow states. Z. Angew. Math. Phys. 36, 854-889.

KOPECKY, R. M. \& TORRANCE, K. E. 1973 Initiation and structure of axisymmetric eddies in a rotating stream. Computers Fluids 1, 289-300.

Krause, E., Shi, X.-G. \& Hartwich, P.-M. 1983 Computation of leading edge vortices. AIAA $\theta^{\text {th }}$ Computational Fluid Dynamics Conf. CP834.

LoPEz, J. M. 1986 Numerical simulation of axisymmetric vortex breakdown. Proc. $9^{\text {th }}$ Australasian Fluid Mech. Conf., Univ. of Auckland, New Zealand.

Lopez, J. M. 1988 Axisymmetric vortex breakdown. Part 1. Confined swirling flow. ARL Aero. Rep. 173 AR-004-572.

Lopez, J. M. 1990 Axisymmetric vortex breakdown. Part 1. Confined swirling flow. J. Fluid Mech. 221, 533-552.

Neitzel, G. P. 1988 Streak-line motion during steady and unsteady axisymmetric vortex breakdown. Phys. Fluids 31, 958-960.

SARPKAYA, T. $1971 a$ Vortex breakdown in swirling conical flows. AIAA J.9, 1792-1799.

Sarpkaya, T. $1971 b$ On stationary and travelling vortex breakdowns. J. Fluid Mech. 45, 545-559.

SHI, X.-G. 1985 Numerical simulation of vortex breakdown. Proc. Colloq. on Vortex Breakdown, Feb. 1985, Sonderforschungsbereich 25, 'Wirbelströmungen in der Flugtechnik', RWTH Aachen, pp. 69-80.

Spall, R. E., Gatski, T. B. \& GResch, C. E. 1987 A criterion for vortex breakdown. Phys. Fluids 30, 3434-3440.

Squire, H. B. 1960 Analysis of the 'vortex breakdown' phenomenon. Part 1. Imperial College, Aero. Dep. Rep. 102.

Uchida, S., Nakamura, Y. \& OHSAwa, M. 1985 Experiments on the axisymmetric vortex breakdown in a swirling air flow. Trans. Japan Soc. Aero. Space Sci. 27, 206-216. 